\title{
Checklist de Simuliidae (Insecta, Diptera) do Estado do Mato Grosso do Sul, Brasil
}

\author{
Nayara Karla Zampiva ${ }^{1,2}$ \& Mateus Pepinelli ${ }^{3}$
}

\author{
1.Programa de Pós-Graduação em Entomologia e Conservação da Biodiversidade, Universidade Federal da Grande Dourados, Rod. Itahum-Dourados, Km 12, \\ Unidade II, Dourados-MS, Brasil. \\ 2.Laboratório de Zoologia, Universidade Federal do Mato Grosso do Sul, Campo Grande, MS, Brasil. \\ 3. Department of Natural History, Royal Ontario Museum, Toronto, Canadá. (mateuspepi@yahoo.com.br)
}

Recebido 28 novembro 2016

Aceito 6 fevereiro 2017

DOI: $10.1590 / 1678-4766 e 2017129$

ABSTRACT. Checklist of Simuliidae (Insecta, Diptera) of state of Mato Grosso do Sul, Brazil.This study provides a checklist of Simuliidae species in the state of Mato Grosso do Sul along with information on distribution of species in 47 rivers and streams of the two major river basins in the State: Paraná and Paraguay. Eighteen species were reported to the state, including two new records: Simulium dinellii (Joan, 1912) and Simulium virescens Hamada, Silva \& Pereira, 2012.

KEYWORDS. Black flies, geographical distribution, new records, Biota-MS Program.

RESUMO. Este trabalho apresenta uma listagem de espécies de simulídeos no Mato Grosso do Sul e traz informações sobre a distribuição das espécies em 47 corpos d'água das duas grandes bacias hidrográficas do estado: Paraná e Paraguai. No total foram reportadas 18 espécies para o estado, incluindo dois novos registros: Simulium dinellii (Joan, 1912) e Simulium virescens Hamada, Silva \& Pereira, 2012.

PALAVRAS-CHAVE. Pium, Borrachudo, Distribuição geográfica, Novos registros, Programa Biota-MS.

Simuliidae, conhecidos popularmente como borrachudos, constituem um grupo de dípteros cosmopolita que possui reconhecida importância, devido principalmente aos adultos de algumas espécies serem vetores de doenças, tais como a oncocercose e mansonelose (SHELley \& Coscarón, 2001).

De acordo com AdLer \& CrossKey (2015) existem atualmente 2.177 espécies de Simuliidae válidas (incluindo 12 fósseis) das quais aproximadamente 360 são encontradas na Região Neotropical (CURRIE \& ADLER, 2008), sendo 92 (AdLER \& CrossKey, 2015; HAMAdA et al., 2015) registradas para o Brasil. Três gêneros de Simuliidae estão registrados para o Brasil: Araucnephia Wygodzinsky \& Coscarón, 1973 com duas espécies, Lutzsimulium d'Andreatta \& d'Andreatta, 1947 com quatro espécies e Simulium Latreille, 1802 com 86 espécies. No estado de Mato Grosso do Sul 14 espécies são registradas (EATON et al., 1998; AdLER \& Crosskey, 2015).

As larvas são aquáticas, reofílicas (dependem de correnteza) e filtradoras, alimentando-se de partículas orgânicas finas (FPOM) dissolvidas e suspensas na água (Alencar et al., 2001). São comumente encontradas em corpos d'água com alto teor de oxigênio dissolvido e com variações na quantidade de matéria orgânica (STRIEDER et al., 2002; STRIEDER et al., 2006). As larvas de último estádio constroem um casulo, produzido com seda secretada pelas glândulas salivares, para dar inicio ao estágio de pupação. Já os adultos são terrestres e ambos os sexos podem se alimentar de néctar e somente as fêmeas de algumas espécies são hematófogas, pois necessitam de sangue de vertebrados para completar o desenvolvimento dos ovos. Dependendo da preferência hematofágica, podem ser zoofílicas, ornitofílicas ou antropofílicas (CROSSKEY, 1990; COSCARÓN \& COSCARÓNARIAS, 2007).

A maioria dos registros de ocorrência de Simuliidae para o Mato Grosso do Sul foram realizados por COSCARÓN (1991), Coscarón \& Coscarón-Arias (2007), AdLER \& Crosskey (2015), porém pouco se sabe sobre as localidades, as datas, os coletores das espécies e o material examinado proveniente desse estado. Dessa forma, neste trabalho apresentamos a lista de espécies de Simuliidae registrada e georreferenciada no estado de Mato Grosso do Sul.

\section{MATERIAL E MÉTODOS}

O estado de Mato Grosso do Sul possui uma área de $357.124,962 \mathrm{~km}^{2}$, banhado por duas grandes bacias hidrográficas: (1) do Rio Paraná com uma área total de $169.488,662 \mathrm{~km}^{2}$, a leste, que se destacam os rios: Aporé, Sucuriú, Verde, Brilhante, Pardo, Ivinhema, Amambai e Iguatemi e (2) do Rio Paraguai com área total de 187.636,300 
$\mathrm{km}^{2}$, ao lado oeste, com destaque para os rios Taquari, Miranda, Aquidauana, Negro e Apa (ZEE-MS, 2007).

Para a elaboração dessa lista de espécies foram utilizadas duas fontes de informações, a primeira foi a identificação do material recentemente coletado dentro do projeto "Distribuição de Simuliidae do estado do Mato Grosso do Sul”, no programa Entomologia e Conservação da Biodiversidade (UFGD). As coletas ocorreram em 47 pontos abrangendo as seguintes sub-bacias do Mato Grosso do Sul: Rio Sucuriú, Rio Verde, Amambai, Brilhante, Aquidauana e Apa (Fig. 1, Tab. II) durante os meses de setembro de 2011 a maio de 2012. Todos os pontos foram amostrados apenas uma vez, e os espécimes coletados manualmente em um trecho de 100 metros de cada corpo d'água. A segunda fonte de informação foi a consulta de trabalhos publicados (EATON et al., 1998; HAMAda \& AdLER, 1999; CosCARón \& Coscarón-Arias, 2007; Adler \& Crosskey, 2015). Dada a importância de disponibilizar informações sobre a distribuição das espécies, foram incluídas no checklist todas as localidades de ocorrência dentro do estado.

O material identificado está depositado na coleção entomológica, Museu da Universidade Federal da Grande Dourados (UFGD).

\section{RESULTADOS E DISCUSSÃO}

Foram identificadas um total de 18 espécies de simulídeos, duas delas sendo novas ocorrências para o estado: S. dinellii (Joan, 1912) e S. virescens Hamada,
Silva \& Pereira, 2012 (Tab. I). Entretanto, duas espécies que constam em nossa lista foram sinonimizadas por HERNANDEZ et al. (2007, 2008): Simulium subclavibranchium Lutz, 1910 sinonimizada com Simulium subnigrum Lutz, 1910 e Simulium acarayense Coscarón \& Wygodzinsky, 1972 sinonimizada com Simulium subpallidum Lutz, 1910, respectivamente. No entanto, corroborando GIL-AzEVEDO et al. (2012), consideramos que existem diferenças morfológicas e caracteres que diferenciam tais espécies, e que é necessário a utilização de outras abordagens e ferramentas para elucidar o status taxonômico dessas espécies, antes de ser realizada qualquer proposta de sinonimização.

Dentre os novos registros de espécies para o estado do Mato Grosso do Sul, S. dinellii possui ampla distribuição geográfica com registros nas regiões Sudeste (São Paulo e Rio de Janeiro) e Sul (Santa Catarina) (AdLER \& Crosskey, 2015). Já $S$. virescens foi descrita recentemente por Hamada et al. (2012) com registro único no rio Correntina, na região Oeste da Bahia. Nosso dado amplia os registros de ocorrência da espécie em 5 graus de latitude em sentido sul do Brasil, uma vez que a coletamos no rio Sucuriú, município de Costa Rica, Mato Grosso do Sul, na divisa com o estado de Goiás.

Das espécies registradas, Simulium exiguum Rouband, 1906, S. incrustatum Lutz, 1910, S. oyapockense Floch \& Abonnenc, 1946 são incriminadas como vetores da Onchocerca volvulus Leuckart na região da Amazônia e Roraima (Cerqueira, 1959; Moraes \& Chaves, 1974; Shelley \& Shelley, 1976; Shelley et al., 1997; Shelley \& CosCARón, 2001). SHElley et al. (2000, 2001) estudaram

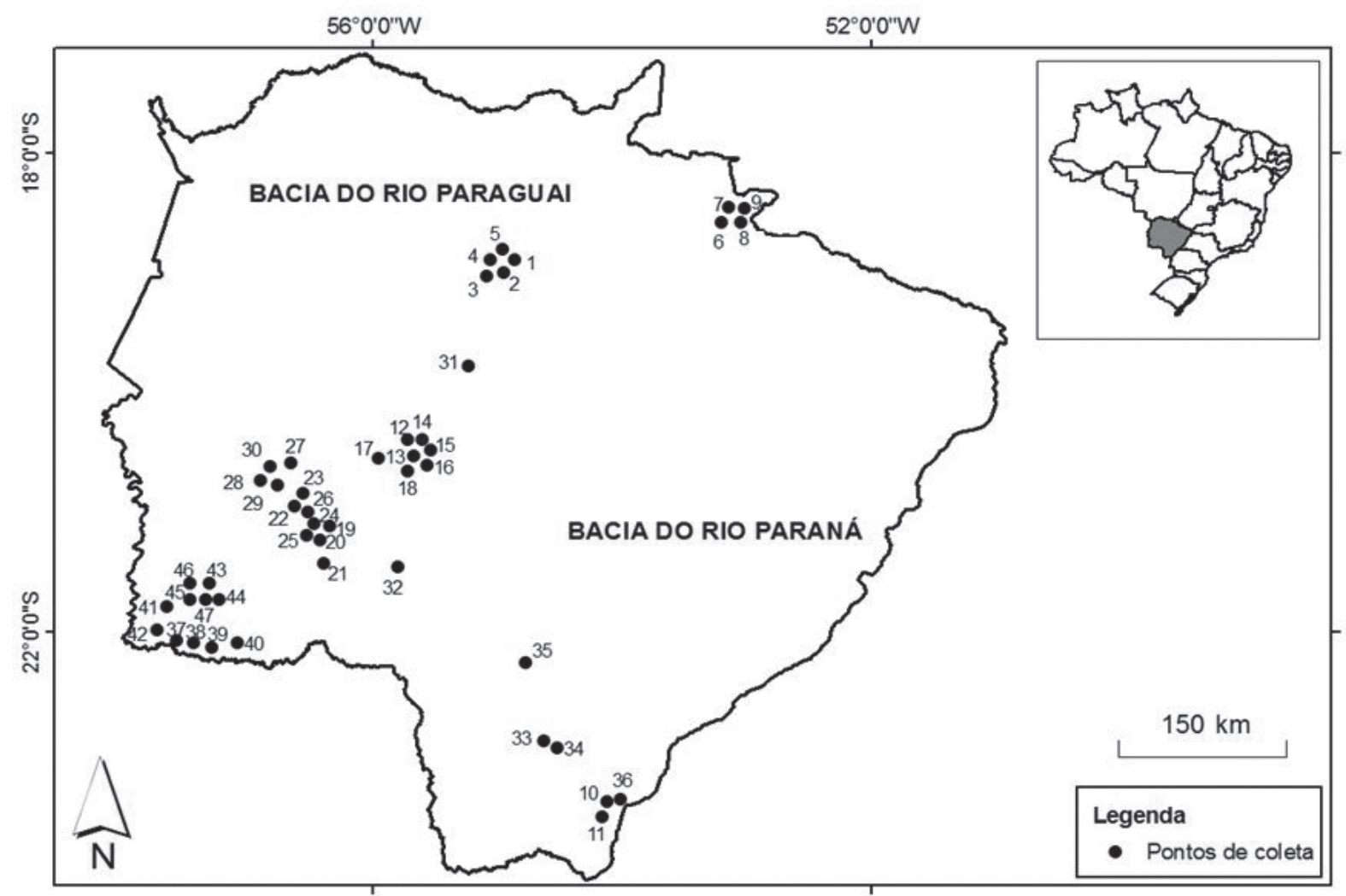

Fig. 1. Mapa dos 47 pontos de coleta de similídeos (Diptera: Simuliidae) no estado do Mato Grosso do Sul, Brasil. 
Tab. I. Lista de espécies de Simulium Latreille, 1802 (Diptera: Simuliidae) do estado de Mato Grosso do Sul, Brasil e localidades de ocorrência (numeração das localidades correspondem àquelas constantes na tabela II) (*, espécie não coletada nesse estudo registrada em ADLER \& CROSSKEY, 2012).

\begin{tabular}{|c|c|c|}
\hline Subgênero & Espécie & Localidade de ocorrência \\
\hline \multicolumn{3}{|l|}{ (Chirostilbia) Enderlein, 1821} \\
\hline & S. dekeyseri Shelley \& Py-Daniel, 1981 & * \\
\hline & S. pertinax Kollar, 1832 & $01,02,03,04,05,06,10,25,33$ \\
\hline & S. spinibranchium Lutz, 1910 & $03,05,07,14,19,30$ \\
\hline & S. subpallidum Lutz, 1910 & $\begin{array}{c}05,07,08,09,12,18,20,21,31,32,33,35,36, \\
39,40,41,42,43,44,45,46,47\end{array}$ \\
\hline \multicolumn{3}{|l|}{ (Hemicnetha) Enderlein, 1934} \\
\hline & S. rubrithorax Lutz, 1909 & $14,15,19,23,28,30$ \\
\hline \multicolumn{3}{|l|}{ (Inaequalium) Coscarón \& Wygodzinsky, 1984} \\
\hline & S. inaequale Paterson \& Shanon, 1927 & $09,29,35,39,44,46,47$ \\
\hline & S. subnigrum Lutz, 1910 & $03,04,05,06,09,12,25,28,29,33$ \\
\hline \multicolumn{3}{|l|}{ (Notolepria) Enderlein, 1930} \\
\hline & S. exiguum Roubaud, 1906 & 07 \\
\hline \multicolumn{3}{|l|}{ (Psaroniocompsa) Enderlein, 1934} \\
\hline & S. incrustatum Lutz, 1910 & 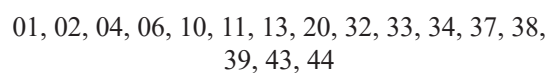 \\
\hline & S. jujuyense Paterson \& Shannon, 1927 & $*$ \\
\hline & S. oyapockense Floch \& Abonnenc, 1946 & 38 \\
\hline & S. siolii Py-Daniel, 1988 & $*$ \\
\hline \multirow[t]{2}{*}{ (Ectemnaspis) Enderlein, 1934} & S. dinellii Joan, 1912 & $06,09,17,37$ \\
\hline & S. perflavum Roubaud, 1906 & $09,35,36,43,46,47$ \\
\hline \multicolumn{3}{|l|}{ (Psilopelmia) Enderlein, 1934} \\
\hline & S. virescens Hamada, Silva \& Pereira, 2012 & 07 \\
\hline \multicolumn{3}{|l|}{ (Trichodagmia) Enderlein, 1934} \\
\hline & S. nigrimanum Macquart, 1983 & $06,11,12,15,16,20,25,32$ \\
\hline \multicolumn{3}{|l|}{ (Thyrsopelma) Enderlein, 1934} \\
\hline & S. hirtipupa Lutz, 1910 & $19,20,22,23,24,25,26,27$ \\
\hline & S. orbitale Lutz, 1910 & $*$ \\
\hline
\end{tabular}

espécies que são vetores potenciais de $O$. volvulus no estado de Goiás e relataram um possível caso de oncocercose no município de Minaçu, no entanto é uma ocorrência isolada e não existem outros registros para a região ou para os estados vizinhos, como o estado de Mato Grosso do Sul. Com relação à S. nigrimanum Macquart, 1983, há indícios de alta incidência de antropofília desta espécie na região foco de Fogo Selvagem na Aldeia Limão Verde, Aquidauana, no Mato Grosso do Sul (EATon et al., 1998). No entanto, ainda é controverso a questão do Pênfigo folíaceo como doença e também quanto à disseminação por simulídeos.

Dentre as espécies coletadas, oito possuem ampla distribuição no estado de Mato Grosso do Sul: Simulium pertinax Kollar, 1832, S. incrustatum, S. subnigrum, $S$. spinibranchium Lutz, 1910, S. subpallidum, S. hirtipupa Lutz, 1910, S. inaequale Paterson \& Shanon, 1927 e S. nigrimanum, com ocorrência na maioria dos pontos amostrados (Tab. I).

$\mathrm{O}$ fato do presente estudo ter aumentado o número de espécies registradas pode ser explicado principalmente pela escassez de estudos realizados no estado de Mato Grosso do Sul. De acordo com AdLer \& Crosskey (2015), no Brasil a Região Sudeste possui o maior número de espécies registradas (58), seguida pelas regiões Sul (44), Norte (42), Centro-Oeste (32) que com este trabalho aumenta para 34 e Nordeste (30). Com os novos registros, o estado de Mato Grosso do Sul passa a ter a riqueza de espécies de Simuliidae semelhante ao estado de Espírito Santo, Amapá e Pará, mas ainda possui poucas espécies em comparação com os estados de Minas
Gerais, Rio de Janeiro e São Paulo, cada um com 35, 37 e 53 espécies respectivamente. Esperamos que com um maior esforço amostral, principalmente em áreas ainda não estudadas ou pouco amostradas, principalmente no centro do estado e no norte, onde está localizada a borda pantaneira (área de transição do Cerrado-Pantanal), aumente ainda mais a diversidade de Simuliidae conhecida para o estado.

Principais grupos de pesquisa e acervos. Dra. Neusa Hamada e Dr. Victor Py-Daniel, do Instituto Nacional de Pesquisas da Amazônia do Instituto Nacional de Pesquisas da Amazônia (INPA); Dra. Marilza Maia-Herzog da FIOCRUZ do Rio de Janeiro; Dr. Mateus Pepinelli, Department of Natural History, Royal Ontario Museum, Canadá; Dr. Leonardo Gil Azevedo, Departamento de Entomologia, Museu Nacional, Universidade Federal do Rio de Janeiro. Destacam-se os seguintes acervos: Instituto Nacional de Pesquisas da Amazônia (INPA); Coleção do Museu de Zoologia da Universidade de São Paulo (USP); Faculdade de Saúde Pública da Universidade de São Paulo (FSP/USP); Instituto Oswaldo Cruz, Fundação Oswaldo Cruz, Rio de Janeiro, Brazil (CSIOC antiga CLSO/IOC); Museu de História Natural de Londres (Department of Entomology, Natural History Museum, London, United Kingdom - BMNH).

Principais lacunas do conhecimento. As principais lacunas do conhecimento citadas por PePINELli (2011) ainda são válidas. Ainda faltam estudos para elucidar as relações evolutivas e os mecanismos de especiação de espécies próximas e isso reflete em uma série de dificuldades 
Tab. II. Corpos d'água amostrados com código de localidades de coletas e coordenadas geográficas, Mato Grosso do Sul, Brasil.

\begin{tabular}{|c|c|c|c|}
\hline Municípios & Corpos d'água & $\begin{array}{c}\text { Cód. Localidades } \\
\text { de Coletas }\end{array}$ & Coordenadas \\
\hline Rio Verde do Mato Grosso & Rio Verde & 1 & $18^{\circ} 56^{\prime} 15.47^{\prime} \mathrm{S}, 4^{\circ} 53 ’ 55.32^{\prime \prime} \mathrm{W}$ \\
\hline Rio Verde do Mato Grosso & Rio Verde & 2 & $18^{\circ} 56^{\prime} 15.3 ” S, 54^{\circ} 54^{\prime} 07.3 ” \mathrm{~W}$ \\
\hline Rio Verde do Mato Grosso & Córrego Cachoeira Bonita & 3 & $18^{\circ} 56^{\prime} 33.3^{\prime \prime} \mathrm{S}, 5^{\circ} 53^{\prime} 54.3^{\prime \prime} \mathrm{W}$ \\
\hline Rio Verde do Mato Grosso & Rio Verde & 4 & $18^{\circ} 56^{\prime} 15.4^{\prime \prime S}, 54^{\circ} 54^{\prime} 55.0^{\prime \prime} \mathrm{W}$ \\
\hline Rio Verde do Mato Grosso & Córrego Sossego & 5 & $18^{\circ} 55^{\prime} 46.3 ” \mathrm{~S}, 54^{\circ} 54^{\prime} 39.8^{\prime \prime} \mathrm{W}$ \\
\hline Costa Rica & Córrego Ribeirão de Baixo & 6 & $18^{\circ} 33^{\prime} 51.13^{\prime \prime S}, 53^{\circ} 7^{\prime} 58.20^{\prime \prime} \mathrm{W}$ \\
\hline Costa Rica & Rio Sucuriú & 7 & $18^{\circ} 26^{\prime} 2.79^{\prime \prime} \mathrm{S}, 53^{\circ} 2^{\prime} 22.54^{\prime \prime} \mathrm{W}$ \\
\hline Costa Rica & Cachoeira da Lage & 8 & $18^{\circ} 31^{\prime} 19.26^{\prime \prime S}, 53^{\circ} 0$ '42.74”W \\
\hline Costa Rica & Córrego Lage & 9 & $18^{\circ} 31^{\prime} 20.44^{\prime \prime S}, 53^{\circ} 0$ '49.84’W \\
\hline Itaquiraí & Córrego São Luiz & 10 & $23^{\circ} 29^{\prime} 55.59^{\prime} \mathrm{S}, 54^{\circ} 4^{\prime} 4.06^{\prime \prime} \mathrm{W}$ \\
\hline Itaquiraí & Córrego Itaquiraizinho & 11 & $23^{\circ} 30^{\prime} 36.5^{\prime \prime} \mathrm{S}, 54^{\circ} 9^{\prime} 16.5^{\prime \prime} \mathrm{W}$ \\
\hline Aquidauana & Córrego Fundo (APP UEMS) & 12 & $20^{\circ} 26^{\prime} 5.4^{\prime \prime} \mathrm{S}, 55^{\circ} 39^{\prime} 33.7^{\prime \prime} \mathrm{W}$ \\
\hline Aquidauana & Rio Aquidauana & 13 & $20^{\circ} 29^{\prime} 1.28^{\prime \prime S}, 55^{\circ} 38^{\prime} 28.92^{\prime \prime} \mathrm{W}$ \\
\hline Aquidauana & Córrego Morcego & 14 & $20^{\circ} 27^{\prime} 3.28^{\prime \prime} \mathrm{S}, 55^{\circ} 37^{\prime} 18.49^{\prime \prime} \mathrm{W}$ \\
\hline Aquidauana & Córrego Paxixi & 15 & 20²9’25.9”S, 55³4’25.6”W \\
\hline Aquidauana & Córrego das Antas & 16 & 20²9’14.1’S, 55॰33’26.4’W \\
\hline Aquidauana & Córrego Acogo & 17 & $20^{\circ} 30^{\prime} 15.8^{\prime \prime} \mathrm{S}, 55^{\circ} 50^{\prime} 58.4^{\prime \prime} \mathrm{W}$ \\
\hline Aquidauana & Córrego Boeiro & 18 & $20^{\circ} 30^{\prime} 33.2^{\prime \prime} \mathrm{S}, 55^{\circ} 39^{\prime} 27.3^{\prime \prime} \mathrm{W}$ \\
\hline Bonito & Rio Formoso & 19 & $21^{\circ} 9^{\prime} 30.4^{\prime \prime} \mathrm{S}, 56^{\circ} 25^{\prime} 29^{\prime \prime} \mathrm{W}$ \\
\hline Bonito & Rio Formosinho & 20 & $21^{\circ} 10^{\prime} 2.45^{\prime \prime} \mathrm{S}, 56^{\circ} 26^{\prime} 15.86^{\prime \prime} \mathrm{W}$ \\
\hline Jardim & Rio da Prata & 21 & $21^{\circ} 25^{\prime} 6.12^{\prime \prime} \mathrm{S}, 56^{\circ} 23^{\prime} 25.19^{\prime \prime} \mathrm{W}$ \\
\hline Bonito & Córrego Chapeninha & 22 & $20^{\circ} 50 ’ 33.2 ” S, 56^{\circ} 35^{\prime} 25^{\prime \prime} \mathrm{W}$ \\
\hline Bonito & Rio Chapena & 23 & $20^{\circ} 49^{\prime} 57.8^{\prime \prime S}, 56^{\circ} 33^{\prime} 12.2^{\prime \prime} \mathrm{W}$ \\
\hline Bonito & Rio Formoso & 24 & $21^{\circ} 8^{\prime} 22.75^{\prime \prime S}, 56^{\circ} 27^{\prime} 5.10^{\prime \prime} \mathrm{W}$ \\
\hline Bonito & Córrego Anhumas & 25 & $21^{\circ} 11^{\prime} 21^{\prime \prime} \mathrm{S}, 56^{\circ} 31^{\prime} 31.1^{\prime \prime} \mathrm{W}$ \\
\hline Bonito & Rio Mimoso & 26 & $20^{\circ} 59^{\prime} 17.8^{\prime \prime S}, 56^{\circ} 30^{\prime} 44.6^{\prime \prime} \mathrm{W}$ \\
\hline Bodoquena & Rio Betione & 27 & $20^{\circ} 34^{\prime} 56.28^{\prime \prime} \mathrm{S}, 56^{\circ} 39^{\prime} 4.46^{\prime \prime} \mathrm{W}$ \\
\hline Bodoquena & Córrego Gruta do Beija-flor & 28 & $20^{\circ} 41^{\prime} 47.5{ }^{\prime \prime} \mathrm{S}, 56^{\circ} 51^{\prime} 54.04$ 'W \\
\hline Bodoquena & Córrego afluente Ouro Fino & 29 & $20^{\circ} 43^{\prime} 49.07^{\prime \prime} \mathrm{S}, 56^{\circ} 51^{\prime} 2.14^{\prime \prime} \mathrm{W}$ \\
\hline Bodoquena & Córrego da Caverna & 30 & $20^{\circ} 42^{\prime} 15.4 ” \mathrm{~S}, 56^{\circ} 50^{\prime} 59.00^{\prime \prime} \mathrm{W}$ \\
\hline Taboco & Córrego Gualheiros & 31 & $19^{\circ} 46^{\prime} 24.3^{\prime \prime S}, 55^{\circ} 13^{\prime} 59.78^{\prime \prime} \mathrm{W}$ \\
\hline Maracajú & Córrego Canindé & 32 & $21^{\circ} 27^{\prime} 1.21^{\prime \prime} \mathrm{S}, 55^{\circ} 47^{\prime} 44.65^{\prime \prime} \mathrm{W}$ \\
\hline Jutí & Córrego Toco Seco & 33 & $22^{\circ} 55^{\prime} 31.04^{\prime \prime} \mathrm{S}, 54^{\circ} 30^{\prime} 10.8^{\prime \prime} \mathrm{W}$ \\
\hline Jutí & Rio Bonito & 34 & $22^{\circ} 53^{\prime} 54.2^{\prime \prime} \mathrm{S}, 54^{\circ} 33^{\prime} 33.13^{\prime \prime} \mathrm{W}$ \\
\hline Dourados & Pesqueiro Kanoas & 35 & $22^{\circ} 14^{\prime} 51.9^{\prime \prime} \mathrm{S}, 54^{\circ} 45^{\prime} 59.26^{\prime \prime} \mathrm{W}$ \\
\hline Itaquiraí & Córrego Salvador & 36 & $23^{\circ} 27^{\prime} 33.78^{\prime \prime} \mathrm{S}, 54^{\circ} 0 ’ 1.47^{\prime \prime} \mathrm{W}$ \\
\hline Porto Murtinho & Córrego Afluente do APA & 37 & $22^{\circ} 10^{\prime} 5.30^{\prime \prime} \mathrm{S}, 57^{\circ} 31^{\prime} 12.70^{\prime \prime} \mathrm{W}$ \\
\hline Porto Murtinho & Cachoeira do APA & 38 & $22^{\circ} 10^{\prime} 18.47^{\prime \prime} \mathrm{S}, 57^{\circ} 31^{\prime} 4.36^{\prime \prime} \mathrm{W}$ \\
\hline Porto Murtinho & Córrego Binguela & 39 & $22^{\circ} 9^{\prime} 49.09^{\prime \prime} \mathrm{S}, 57^{\circ} 31^{\prime} 34.70^{\prime \prime} \mathrm{W}$ \\
\hline Porto Murtinho & Córrego Jango Fundo & 40 & $22^{\circ} 4^{\prime} 59.14^{\prime \prime} \mathrm{S}, 57^{\circ} 14^{\prime} 40.3^{\prime \prime} \mathrm{W}$ \\
\hline Porto Murtinho & Córrego Mamonal & 41 & $22^{\circ} 0 ’ 4.10^{\prime \prime S}, 57^{\circ} 34^{\prime} 24.80^{\prime \prime} \mathrm{W}$ \\
\hline Porto Murtinho & Córrego Lapiá & 42 & $21^{\circ} 57^{\prime} 12.7^{\prime \prime} \mathrm{S}, 57^{\circ} 35^{\prime} 55.7^{\prime \prime} \mathrm{W}$ \\
\hline Porto Murtinho & Córrego Piquenique & 43 & $21^{\circ} 38^{\prime} 09^{\prime \prime S}, 57^{\circ} 18^{\prime} 07^{\prime \prime} \mathrm{W}$ \\
\hline Porto Murtinho & Córrego Três Canos & 44 & $21^{\circ} 40^{\prime} 30.6^{\prime} \mathrm{S}, 57^{\circ} 17^{\prime} 44.7^{\prime \prime} \mathrm{W}$ \\
\hline Porto Murtinho & Córrego Cabrito & 45 & $21^{\circ} 42^{\prime} 20.5^{\prime \prime} \mathrm{S}, 57^{\circ} 25^{\prime} 27.91^{\prime \prime} \mathrm{W}$ \\
\hline Porto Murtinho & Córrego Dobrado & 46 & $21^{\circ} 42^{\prime} 23^{\prime \prime} \mathrm{S}, 57^{\circ} 25^{\prime} 49.34^{\prime \prime} \mathrm{W}$ \\
\hline Porto Murtinho & Córrego Triste & 47 & $21^{\circ} 41^{\prime} 29.6^{\prime \prime} \mathrm{S}, 57^{\circ} 23^{\prime} 47.61^{\prime \prime} \mathrm{W}$ \\
\hline
\end{tabular}

para identificação e classificação de Simuliidae no Brasil. Somente uma abordagem múltipla, com uso de marcadores moleculares, cromossomos politênicos, morfologia, ferramentas morfométricas, entre outros, poderá elucidar a história evolutiva de espécies próximas, que muitas vezes são tratadas como complexos de espécies, ou sinonimizadas baseadas em carateres morfológicos altamente varíaveis.

Perspectivas de pesquisa para o grupo nos próximos 10 anos. Necessidade de formação de jovens especialistas no grupo. É fundamental que os pesquisadores de Simuliidae no Brasil cheguem a um consenso com relação à classificação do grupo e, para isso, serão necessários estudos filogenéticos mais completos, com uso de vários marcadores moleculares e caracteres morfológicos. Simuliidae é um grupo com elevado potencial para o biomonitoramento e existe uma grande perspectiva de pesquisa para utilização de espécies de Simuliidae para índices bióticos de qualidade ambiental e da água de córregos e rios.

Agradecimentos. A Fundação de Apoio ao Desenvolvimento do Ensino, Ciências e Tecnologia do Estado de Mato Grosso do Sul (Fundect) e a Superintendência de Ciências e Tecnologia do Estado de Mato Grosso do Sul (Sucitec/MS) pelo convite de participação neste fascículo especial da Iheringia Série Zoologia e o suporte financeiro para sua publicação. 


\section{REFERÊNCIAS BIBLIOGRÁFICAS}

Adler, P. H. \& Crosskey, R. W. 2015. World Black Flies (Diptera: Simuliidae): a comprehensive revision of the taxonomic and geographical inventory. Disponível em $<\mathrm{http}$ ://entweb.clemson. edu/biomia/pdfs/blackflyinventory.pdf > . Acessado em 27.04.2015.

Adler, P. H.; Currie, D. C. \& Wood, D. M. 2004. The Black Flies (Simuliidae) of North America. Ithaca, Cornell University Press. 941p.

Alencar, Y. B.; Ludwig, T. A. V.; Soares, C. C. \& Hamada, N. 2001. Stomach content analyses of Simulium perflavum Roubaud 1906 (Diptera: Simuliidae) larvae from streams in Central Amazônia, Brazil. Memórias do Instituto Oswaldo Cruz 96:561-576.

CerqueIRa, N. L. 1959. Sobre a transmissão de Mansonella ozzardi (I e II notas). Jornal Brasileiro de Medicina 1: 885-914.

Coscarón, S. 1991. Fauna de água dulce de la República Argentina. Insecta, Diptera, Simuliidae. FECIC, Buenos. Aires, Argentina.

Coscarón, S. \& Coscarón-Arias, C. L. 2007. Neotropical Simuliidae (Diptera: Simuliidae). In: AdIS, J.; ARIAS, J. R.; Rueda-Delgado, G. \& Wantzen, K. M. eds. Aquatic Biodiversity in Latin America (ABLA). Vol.3. Sofia-Moscow, Pensoft. 685p.

Crosskey, R. W. 1990. The Natural History of Blackflies. London, The British Museum of Natural History. 711p.

Eaton, D. P.; Diaz, L. A.; Hans-Filho, G.; Santos, V.; Aoki, V.; Friedman, H.; Rivitti, E. A.; Sampaio, S. A. P.; Gottlieb, M. S.; Giudice, G. J.; Lopez, A.; Cupp, E. W. \& The Cooperative Group on Fogo Selvagem RESEARCH. 1998. Comparison of black fly species (Diptera: Simuliidae) on an Amerindian reservation with a high prevalence of Fogo Selvagem to neighboring disease-free sites in the state of Mato Grosso do Sul, Brazil. Journal of Medical Entomology 35(2):120-131.

Gil-Azevedo, L. H.; Coscarón, S. \& Maia-Herzog, M. 2012. The phylogeny of Simulium (Chirostilbia) (Diptera: Simuliidae) and perspectives on the systematics of the genus in the Neotropical Region. Memórias do Instituto Oswaldo Cruz 107(2):178-185.

Hamada, N. \& AdleR, P. H. 1999. Cytotaxonomy of four species in the Simulium perflavum species group (Diptera: Simuliidae) from Brazilian Amazonia. Systematic Entomology 24:273-288.

Hamada, N.; Silva, N. G. \& Pereira, E. S. 2012. Simulium (Psilopelmia) virescens, a new black-fly species (Diptera: Simuliidae) from the southwestern region of the state of Bahia, Brazil. Memórias do Instituto Oswaldo Cruz 107(1):102-110.

Hamada, N.; Nascimento, J. M. C. \& Pepinelli, M. 2015. A new species of Simulium (Chirostilbia) (Diptera: Simuliidae) from Mantiqueira mountain range, southeastern Brazil. Acta Tropica 150:143-158.

Hernández, L. M.; Shelley, A. J.; Luna-Dias, A. P. A. \& MaiA-Herzog, M. 2007. Review of the Neotropical blackfly subgenus Inaequalium
Coscarón \& Wygodzinsky (Diptera: Simuliidae) based on adults and pupal morphology. Zootaxa 1649:1-96.

Hernández, L. M.; Shelley, A. J.; Luna-Dias, A. P. A. \& MaiA-Herzog, M. 2008. Review of the Neotropical blackfly subgenus Chirostilbia Enderlein (Diptera: Simuliidae) based on adults and pupal morphology. Zootaxa 1834:1-100.

Moraes, M. A. P. \& Chaves, G. M. 1974. Oncocercose no Brasil. Novos achados entre os índios Ynanomamas. Boletín de la Oficina Sanitária Panamericana 76:48-54.

Pepinelli, M. 2011. Checklist de Simuliidae (Insecta, Diptera) do Estado de São Paulo, Brasil. Biota Neotropical 11(1a). Disponível em $<\mathrm{http}: / /$ www.biotaneotropica.org.br/v11n1a/en/abstract?inventory+bn034110 1a2011>. Acessado em 5.5.2013.

Shelley, A. J. \& Coscarón, S. 2001. Simuliid Blackflies (Diptera: Simuliidae) and ceratopogonid midges (Diptera: Ceratopogonidae) as vectors of Mansonella ozzardi (Nematoda: Onchocercidae) in northern Argentina. Memórias do Instituto Oswaldo Cruz 96(4):451-458.

Shelley, A. J.; Lowry, C. A.; Maia-Herzog, M.; Luna-Dias, A. P. A. \& Moraes, M. A. P. 1997. Biosystematic studies on the Simuliidae (Diptera) of the Amazonia onchocerciasis focus. Bulletin of the Natural History Museum 66(1):1-120.

Shelley, A. J.; Luna-Dias, A. P. A.; Maia-Herzog, M.; Lowry, C. A.; Guarritano, P. R.; Penn, M. \& Camargo, M. 2001. Simiulim cuasiexiguum, a new blackfly species (Diptera: Simuliidae) from the Minaçu Area in the State of Goiás, Central Brazil. Memórias do Instituto Oswaldo Cruz 96:483-496.

Shelley, A. J.; Maia-Herzog, M.; Lowry, C. A.; Luna-Dias, A. P. A.; Guarritano, P. R.; Shelley, A.; Camargo, M. \& Carter, H. G. 2000. The Simuliidae (Diptera) of the secondary onchocerciasis focus at Minaçu in central Brazil. Bulletin of Natural History Museum, Entomology ser. 69:171-221.

Shelley, A. J. \& Shelley, A. 1976. Further evidence for the transmission of Mansonella ozzardi by Simulium amazonicum in Brazil. Annals of Tropical Medicine and Parasitology 70(2):213-217.

Strieder, M. N.; Santos, J. E. \& PÊs, A. M. O. 2002. Diversidade e distribuição de Simuliidae (Diptera, Nematocera) no gradiente longitudinal da bacia do rio dos Sinos, no Rio Grande do Sul, Brasil. Entomología y Vectores 9(4):527-540.

Strieder, M. N.; SAntos, J. E. \& Vieira, E. M. 2006. Distribuição, abundância e diversidade de Simuliidae (Diptera) em uma bacia hidrográfica impactada no sul do Brasil. Revista Brasileira de Entomologia 50(1):119-124.

ZEE-MS - Zoneamento EcolóGico-Econômico do Mato Grosso do Sul. 2007. Contribuições Técnicas, Teóricas, Jurídicas e Metodológicas. Vol. I-III. Disponível em <http://www.semac.ms.gov.br/zeems>. Acessado em 30.11.2012. 